\title{
Deep Learning Based Multi-Modal Biometric Security System Using Visible Light Communication
}

\author{
ARTHI.R, MANOJKUMAR.D, AKSA ABRAHAM, ALLADA RAHUL KISHAN, ALEKHYA \\ SATTENAPALLI \\ Department of Electronics and Communication Engineering \\ SRM Institute of Science and Technology, \\ Ramapuram Campus, Chennai. \\ INDIA
}

\begin{abstract}
Multi-biometric system is an advanced technology which has a wide application space in the field of information security. This work proposes the design of a personal identification system based on a combination of biometric inputs such as face, finger vein, iris and fingerprint. Viola jones algorithm is used for face detection. Convolutional neural network (CNN) with different optimisers are used to steeply raise the image texture and extract high definition distinct features of the input images. The deep dream image algorithm accompanies CNN by visualizing these images and by highlighting the image features learnt by the network. These images are used for understanding and diagnosing network behaviour. This network obtains a high recognition rate, which proves to be better performing than traditional algorithms. In addition to these, a high-speed advanced wireless communication technology (Li-Fi) is used in combination with GSM which would act as an alert system that effectively helps in notifying the supervisory authority, if the system is being trespassed without proper authentication.
\end{abstract}

Key-Words: - Multi-biometric, Convolutional Neural Network (CNN), Viola Jones Algorithm, Deep Dream Image Algorithm, SGDM, RMS Prop, Adam, Light Fidelity (Li-Fi)

Received: March 10, 2021. Revised: October 16, 2021. Accepted: December 12, 2021. Published: January 7, 2022.

\section{Introduction}

Biometrics contribute significantly in the field of information security and plays a cardinal role in the area of authentication and access control. Biometric is metrics concomitant with human attributes. Biometric authentication is a mark of identification [1] and has many applications for personal and industrial needs. Biometric authentication is an encapsulation of biometric identifiers and high-end processing algorithms. Biometric identifiers are classified into various characteristics. A multibiometric system is an advanced technology that fuses various biometrics [2] or different sets of the same biometrics in order to tighten the security and prevent the system from spoof attacks. Multibiometric systems have a higher rate of security [3] when compared to unimodal biometric systems. The fusion of the different biometrics or different sets of same biometric takes place in different stages of the processing stage. These algorithms strengthen the core of entire system.

Various algorithms are used for processing the biometric input. $\mathrm{CNN}$ is one of the most enhanced and highly accurate algorithms that has a higher rate of recognition and accuracy. It works on the principle of biological visual cortex. The neurons in brain is the study pattern and origin sets the base of this algorithm. These neural networks are used in various arenas of a processing stage such as object detection, image classification, image enhancement, etc. CNN has a specific workflow that includes loading the data, analyzing the data, data preprocessing, creating the network, modelling the data, training the model, model evaluation of the test set, predicting labels and finally creating the classification report. Once the image input has been processed and matched, the processed and matched input [4] were mapped to the user specific application or the industry specific application.

Biometrics has a wide space of applications that includes specific and combined applications [5]. Data transmission plays a vital role [6] in various varieties of application based needs. GSM is one of the most common means of transmission. The security breach has been notified via sending a message to the 
authorized person using GSM.

The distinct characteristics of this paper can be concluded in three-fold: Firstly, we propose a multibiometric system using four physiological biometric identifiers namely face, finger vein, iris and fingerprint. Viola jones algorithm has been used for face detection. Secondly, the input images are processed using convolutional neural network algorithms. The mechanism of occlusion has been used to adjust the parameters and also the number of layers in the network. The deep dream algorithm accompanies $\mathrm{CNN}$ by visualizing these images and by highlighting the image features learnt by the network. These images are used for understanding and diagnosing network behavior. Thirdly, high speed advanced wireless communication technology (Li-Fi) is used in combination with GSM and an alert system which effectively helps in notifying the supervisory authority, if the system is being trespassed without proper authentication.

Section 1 contains the Introduction. Section 2 gives a list of all the related works that is used in this paper to make it more informative. Section 3 contains the System Architecture and the usage of components that has been used in the following system. Section 4 discusses about the Results where an overall experience on the possibilities of the various optimizers used and the alert system is given. Section 5 concludes and discusses about the future work.

\section{Related Work}

A new multi-biometric system with many traits such as face, voice and iris are integrated [7] with a multimodel biometric system to overcome the limitations of the unimodal biometric system. The ROC curves of three-single biometric were plotted to compare the accuracy rate of each system. The result obtained from the ROC curves states that the multi-model system provides better accuracy than the individual system.

From [8], the authors had proposed a new concept in the year 2018 that is CNN for extracting the features and identifying the user. The authors observed that in the field of authentication of biometrics, CNN produces a result with roughly $100 \%$ accuracy rate. The layers which have the capacity to produce high quality results are fully connected and Convolutional. From [9] various biometric systems are compared. CNN does the extraction of features. According to the author, the multi-model gives a result of high accuracy along with excellent performance as compared to the unimodal. It has also determined the score level fusion gives $100 \%$ accuracy than feature level fusion that gives only a rate of $99.22 \%$.

From [10] the training on fractionally occluded images is reduced. A training process is proposed to reduce the spatial region of the filters. Three types of regularizations are considered. From [11], [12] and [13], the optical wireless transmission of data has been proposed and LED's were used. It was proved that the data rate is effective, and also provides an alternate solution for the radio frequency spectrum crisis.

\section{System Architecture}

The complete System Architecture has been divided into three parts. In the first part, the input images of face, finger vein, iris and fingerprint are obtained using the biometric devices and the images are preprocessed, cropped, resized, and integrated with CNN. In the second part, three different optimizers are used and they are varied with each simulation to obtain the most efficient optimizer. Changes are made to the hyper parameters that calculate the loss and gain accordingly.

In the third part, high speed advanced wireless communication technology (Li-Fi) has been used in combination with GSM and an alert system which effectively helps in notifying the supervisory authority, if the system is being trespassed without proper authentication. The process of the system considers the input image of the face, finger vein, iris and fingerprint biometric has been taken with the help of biometric devices respectively. The Proposed System Architecture of multi-biometric Transmitter is as shown in Fig.1. and Receiver with an alert system is as shown in Fig.2.

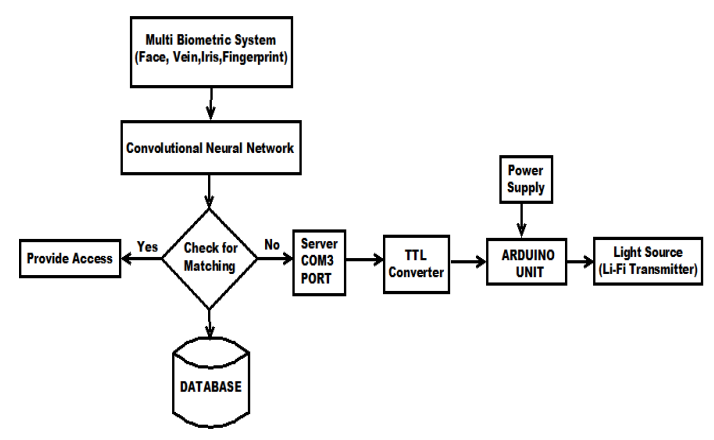


Fig. 1. Architecture of Multi-Biometric Transmitter

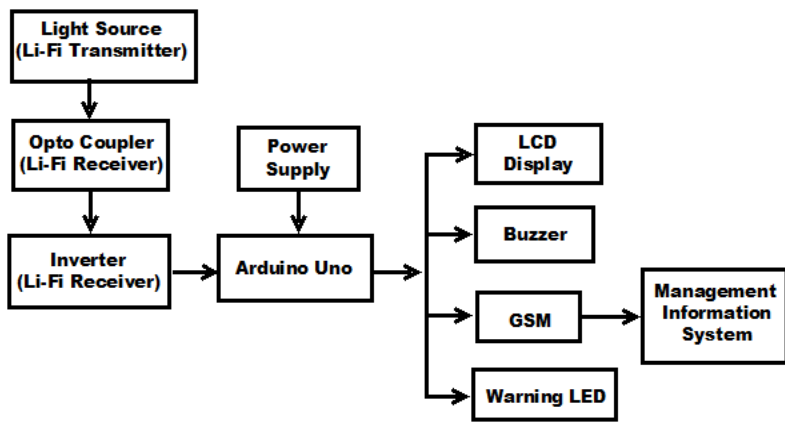

Fig. 2. Architecture of Multi-Biometric Receiver with an alert system.

The input image after getting preprocessed [14] gets converted from RGB to gray to make extraction easier as it doesn't have any color information. The input image is preprocessed, converted and operated by $\mathrm{CNN}$ and later is processed by the deep dream image algorithm which highlights the important features of the image. This highlighted image has been passed to the layers in $\mathrm{CNN}$. The training of CNN was done with three different optimizers, namely: SGDM, RMSPROP, ADAM for comparison of the best system in terms of speed, accuracy, etc. Occlusion has been applied to obtain the exact feature that the network is looking for. These processed trained input images are matched with biometric images of the authorized person from the database and thereby the results are obtained. While matching takes place, the system has been subdivided into two parts based upon the results of matching. If matching takes place successfully for all the mentioned biometrics, the person was given access to the system. Else, the system passes through the security control that triggers the Li-Fi and GSM present in the system. Li-Fi helps in data transmission and through GSM the message is sent to the concerned authority, if the security breach takes place.

\subsection{Deep Dream Image Algorithm}

Deep Dream Image Algorithm is a visualization technique that deals with the features of a specimen by processing an image in the network layer. The algorithm uses gradient ascent [15] [16] [17] [18] that is set equal to the activations from that layer done on the image. Therefore, this maximizes the activations of that layer. The way deep dream algorithm can be used is:

(1) The given input image is passed to the deep dream algorithm and the network is trained along with back propagation to modify the input image in turn to diminish categorization errors.

(2) When the image input has been given into the network, this algorithm utilizes to emphasize the essential features in the specified input image. These layers can extract out those features exactly rather than focusing on the other features.

The important advantage with the latter one is that the weights are not mandatory to be changed when the classification error occurs. Instead of varying the weights of the network, it can also be rectified by highlighting the other features which are not highlighted before. Therefore, the proposed work prefers to go with the latter use.

\subsection{CNN Architecture}

The proposed system architecture consists of an input layer, 3 convolution layers, namely (conv1, conv2, conv3), 1 fully connected layer. The parameters of the network are as shown in Table 1. Table 2. shows the various optimizers which were used and also the hyper parameters which were tuned precisely to avoid the problem of overfitting and under fitting.

Table 1. Layers of ConvNet and Parameters

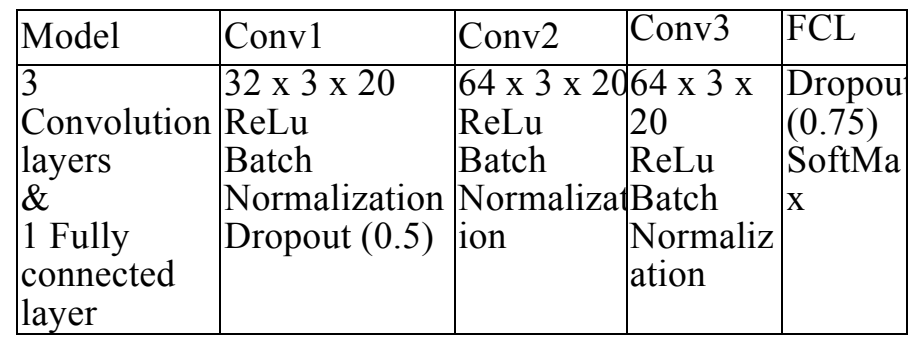

Table 2. Optimizers used and its hyper parameters

\begin{tabular}{|c|c|}
\hline Optimizers & Hyper parameters \\
\hline $\begin{array}{ll} & \text { SGDM } \\
- & \text { RMS Pror } \\
- & \text { Adam }\end{array}$ & $\begin{array}{ll}- & \text { Momentum: } 0.9000 \\
- & \text { Initial Learning Rate: Varies } \\
\text { - } & \text { L2 Regularization: } 1.0000 \mathrm{e}-04 \\
\text { - } & \text { Max Epochs: } 20 \\
\text { - } & \text { Mini Batch Size: } 64 \\
\text { - } & \text { Verbose: } 1 \\
\text { - } & \text { Validation Frequency: } 50 \\
\text { Validation Patience: }: \text { Inf }\end{array}$ \\
\hline
\end{tabular}

\section{Results and Discussions}

The experimental outcome discussed in the 
proposed work gives clarity about the feature extraction at different layers, Mini batch accuracy over the Learning rate and activation strength variations for iteration level.

\subsection{Activations of CNN layers}

The inner processing of all visible layers for all the four biometrics is obtained. The input image is taken and it is converted to the binary image to avoid inter class variations as shown in Fig.3., Fig.4., Fig.5., and Fig.6. The input image is passed to convolution layer to extract the important features that are necessary. The ReLu activation is then applied to the image to increase the non-linearity thereby removing negative values from the image.

As seen, Max pooling layers are dark in nature to grab maximum value in the image and also to avoid the area that are not the features of the images. By this, the parameters are reduced and therefore the model won't over fit on that information. The desired classes are quantized in this layer. After the processing of the image by this layer, the image is classified on the basis of SoftMax classifier and the matching output has been displayed.

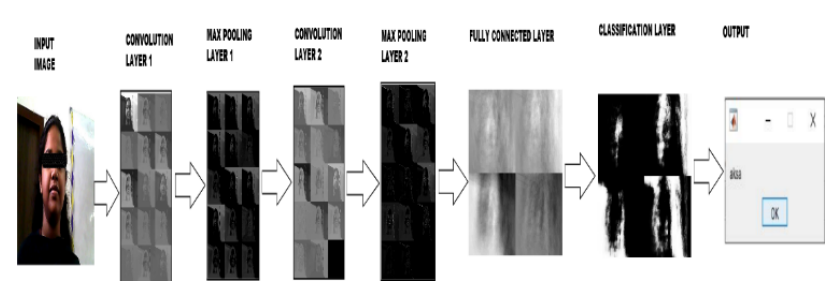

Fig. 3. Activations of Face input at different layers of the network

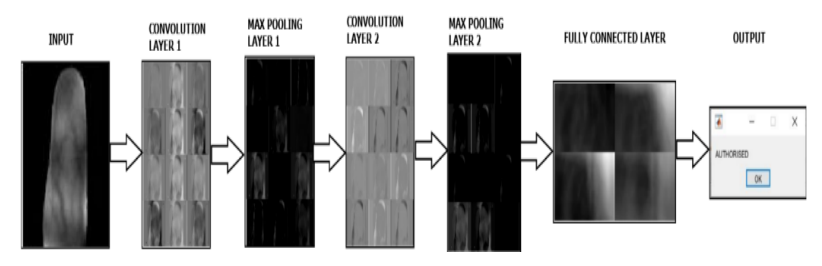

Fig. 4. Activations of Finger vein input at various layers of the network

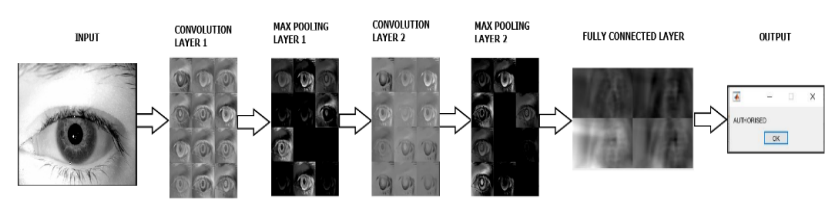

Fig. 5. Activations of Iris input at various layers of the network

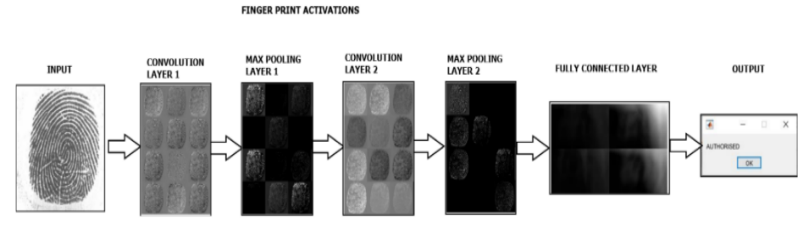

Fig. 6. Activations of Finger print input at different layers of the network

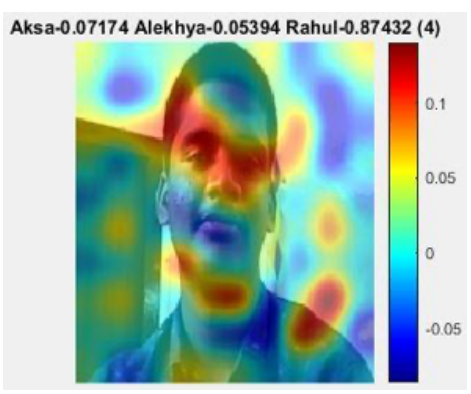

Fig. 7. Occlusion Result of the image after brute force approach

The image obtained from the occlusion is obtained and is shown above. The probability of all the three classes are obtained as shown in the Fig.7. After adjusting the parameters and number of layers in the network with help of the previous image obtained from occlusion mechanism, we could see that the network now obtains the important features from the region of eyes.

\subsection{CNN Architecture}

Fig.8, Fig.9, Fig.10 and Fig.11. shows the efficiency of three different optimizers, namely SGDM, Adam and RMS prop plotted between learning rate and mini batch accuracy. The comparison between these optimizers [19] obtained represents the most efficient and accurate optimizer for different input images.

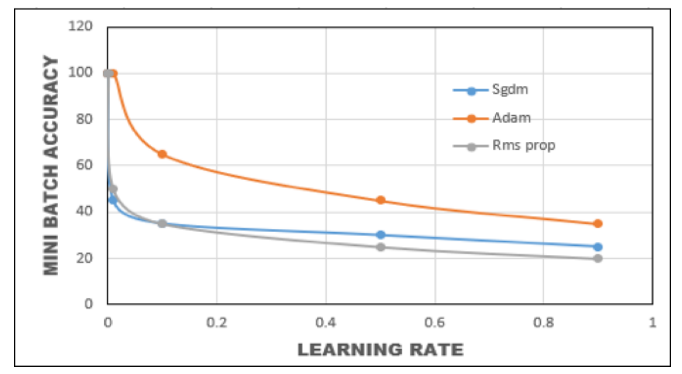

Fig. 8. Performance of Face Biometric 


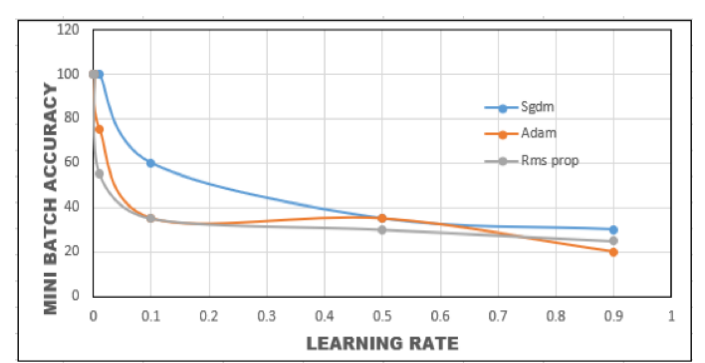

Fig. 9. Performance of Vein Biometric

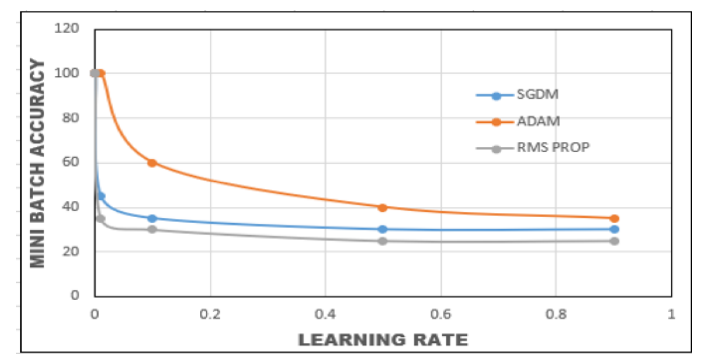

Fig. 10. Performance of Iris Biometric

The input images are trained and iterated more than 20 times. The mini batch size for the training of layers is set as 64 . The layers are trained with the above-said optimizers with different learning rates.

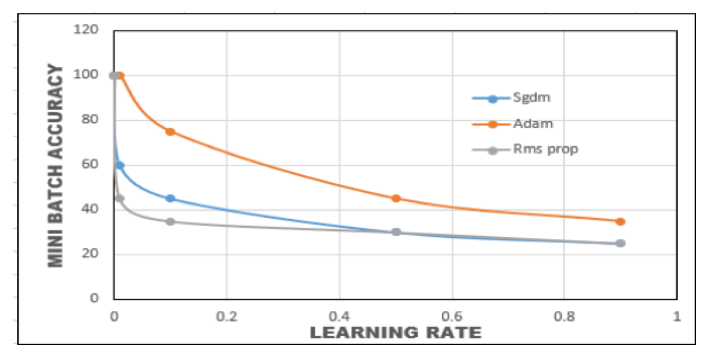

Fig. 11. Performance of Finger Print Biometric

SGDM optimizer gives the peak value of the accuracy rate for finger vein recognition. Adam optimizer gives the peak value of accuracy rate for other biometrics as the mini batch accuracy rate is one hundred-percent for the first three learning rates $(0.0001,0.001$, and 0.01$)$ and gradually decreases for the rest of the learning rates. The other optimizers show a hundred percent accuracy only for the first two learning rates $(0.0001,0.001)$ and shows a steep decrease for the rest of the learning rates. The loss is minimized for Adam optimizer in case of iris, face and finger print biometrics and SGDM optimizer in case of finger vein biometric. The time elapse for finger vein biometric is comparatively lesser than other biometrics.

\subsection{Activation Strength Variations}

The Activation strength was varied with respect to iterations is shown in Fig.12.

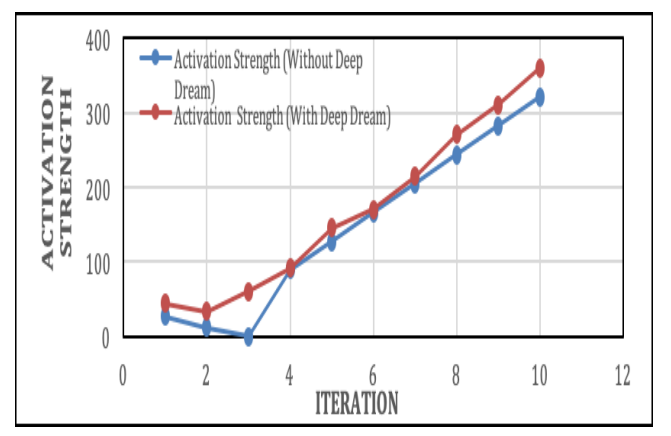

Fig. 12. Graphical representation of activation strength variations

The result was observed with convolution layer 1 . The proposed work clearly infers from the graph that Activation strength with deep dream has been increasing when compared to activation strength without deep dream. It was also observed that the frequency of categorical errors has been very much decreased when using deep dream algorithm.

\subsection{Hardware outputs for alert system}

The hardware part is divided into two parts i.e. Transmitter and Receiver. Fig.13. shows the transmitter which is interfaced with the software part of the system. The serial port connection sends the data to the transmitter when the matching criteria/conditions are not met. Hence, this data is used to trigger the hardware part of the system. From the software part of the system, COM3 port is used to send data through the TTL converter to the Arduino. The Arduino (in transmitter) processes the data received by the Software. This processed data is the sent to the light transmitter which transmits to the receiver part of the $\mathrm{Li}-\mathrm{Fi}$.

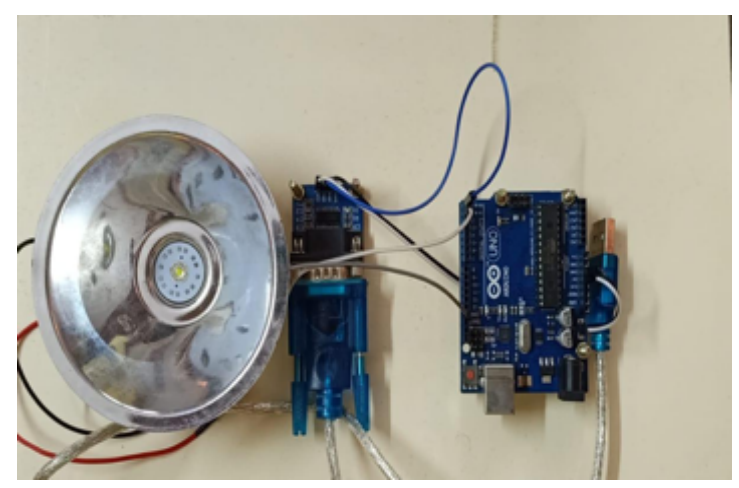

Fig. 13. Li-Fi Transmitter

Fig.14. shows the receiver part which is interfaced with the transmitter part. The receiver part receives 
the data and is then sent to the Arduino (Microcontroller) and then processes this data to trigger LED, buzzer, GSM, and LCD display. LED will glow red light (blinking) indicating this as an

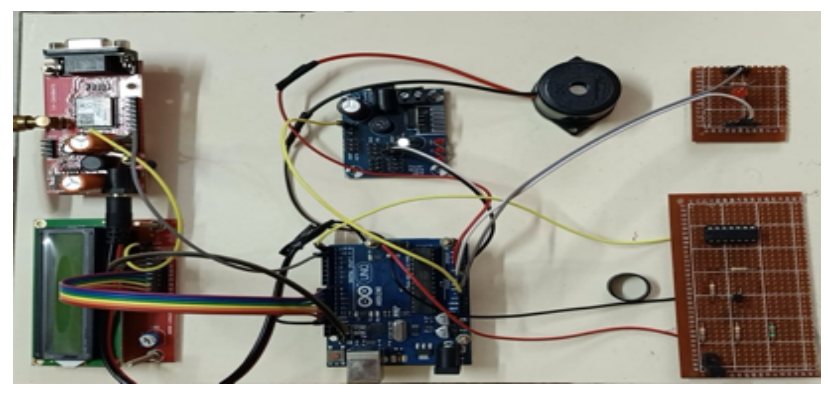

Fig. 14. Li-Fi Receiver

emergency.

The buzzer produces a beep sound to warn. GSM triggers the message immediately to the supervised authority on the system being trespassed. LCD displays on the screen. The combination of all these hardware components together can potentially warn the supervisory authority.

The Fig. 15 and Fig.16 delineates the working of the system. The biometric system transmits the data (in the form of a char) during every authentication. Different char helps us in distinguishing if the access has to be granted or be denied.

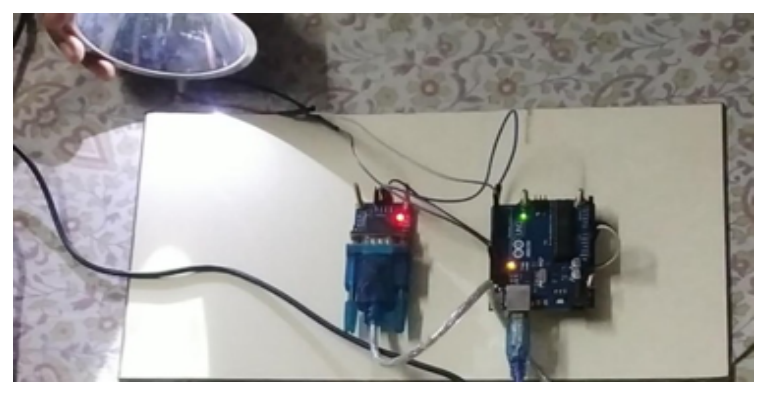

Fig.15.Working of Li-Fi transmitter

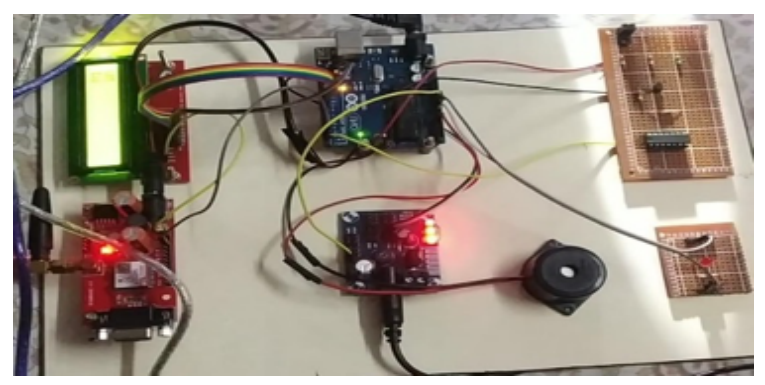

Fig. 16. Working of Li-Fi receiver
The data which is transmitted from biometric system is received at transmitter side. If the data belongs to the category to which access can be granted, the transmitter ends up in giving the access to the system. If the data belongs to the unauthenticated category, the lifi transmitter transmits the data in the form of Light (Li-fi as in Fig.15) to the receiver end as shown in Fig.16.

The receiver then glows up the LED, displays message on LCD, rings a buzzer and also transmits a message with the help of GSM triggered by the Receiver to the possessor of the system.

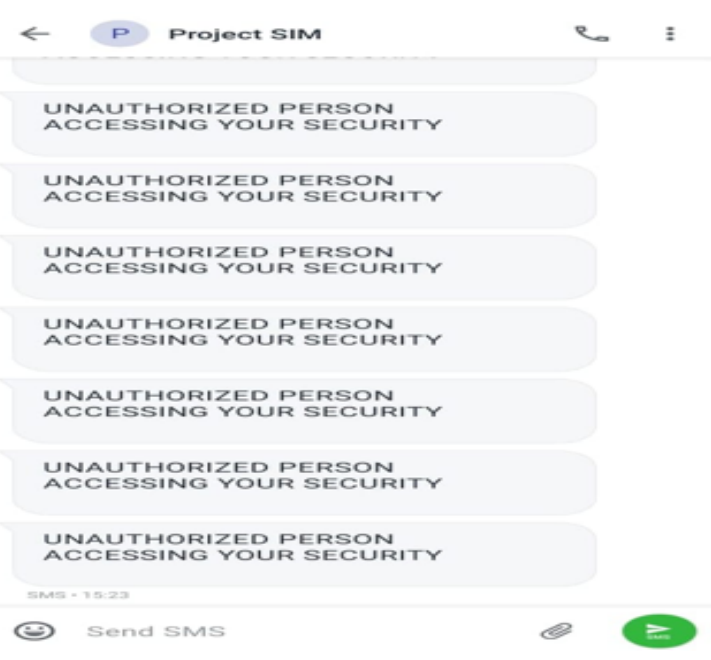

Fig. 17. Message transmitted through GSM

Fig.17. shows the message transmitted by the GSM from the receiver side warning the possessor that some authenticated person is trying to trespass the system.

\section{Conclusion and Future Work}

Combination of various biometrics quiets the concern of security in secured places. The network that the proposed work experimented on contains 3 convolutional layers and 1 fully connected layers. $\mathrm{CNN}$ algorithm has been used for processing the acquired images and for classifying the images. The accurate parameters and layers are used due to the help of the mechanism of occlusion. The categorical errors are reduced with the help of Deep dream image algorithm by improving the activation strength of layers. The accurate tuning of hyper-parameters are done with the help of occlusion mechanism which results in the reduction of overfitting and under fitting problems. $\mathrm{CNN}$ is observed to concentrate only on the necessary features. Three optimizers namely Adam, RMS prop, SGDM has been used for 
simulation and its performance were compared based on learning rate and their accuracy. From the above results, it can be concluded that Adam provides higher stability in the network with respect to increase in learning rates. An alert system is adjoined with the biometric system for warning the security breach and a message has been sent to the authorized person via GSM. In future, Learning rates can be increased therefore the time elapsed for the training could be increased. Many more features can be appended with the existing biometrics. The net can be simulated further by using the other optimizers such as Adagrad, Adadelta, etc. The Grad-CAM can be used in place of occlusion to provide the better tuning of hyper parameters and also to get the better network predictions. Hence, this can reduce the activity bias. Li-Fi can be replaced by any other faster means of communication, which can potentially reduce the line-of-sights problems also.

\section{References:}

[1] R.F Tazim, M. M. M Miah, S. S. Surma, M. T. Islam, C. Shahnaz, \& S. A Fattah, "Biometric Authentication Using CNN Features of Dorsal Vein Pattern Extracted from NIR Image", TENCON 2018-2018 IEEE Region 10 Conference, 2018, pp.19231927.

[2] S Chaudhary, R Nath, "A New Multimodal Biometric Recognition System Integrating Iris, Face and Voice", in International Journal of Advanced Research in Computer Science and Software Engineering,2015, pp $145-50$.

[3] R Devi and P Suiatha, "A Study On Biometric And Multi-Modal Biometric System Modules, Applications, Techniques And Challenges", Conference on Emerging Devices and Smart Systems, 2017, pp. 267271 「ICEDSS1.

[4] S. Albawi, T. A Mohammed, and S. AlZawi, "Understanding of a Convolutional Neural Network" in International Conference on Engineering and Technology, 2017, pp. 1-6 [ICET].

[5] K Lai, S. Samoil, S. N. Yanushkevich, \& G. Collaud, "Application Of Biometric Technologies In Biomedical Systems" in The 10th International Conference on Digital Technologies 2014, pp. 207-216.

[6] M. A Ozkan, \& S. B. Ors, "Data Transmission via GSM Voice Channel for End To End Security", IEEE 5th International Conference on Consumer Electronics-Berlin, 2015, pp. 378-382, 「ICCE-Berlin].

[7] M. Elhoseny, A Elkhateb, A Sahlol, \& A. E Hassanien, "Multimodal Biometric Personal Identification And Verification", Advances in Soft Computing and Machine Learning in Image Processing in Springer, Cham 2018, pp. 249-276.

[8] A Rattani, N. Reddy \& R. Derakhshani, "Multi-Biometric Convolutional Neural Networks for Mobile User Authentication" in IEEE International Symposium on Technologies for Homeland Security HST, pp. 1-6, 2018.

[9] A. S. Al-Waisy, R. Qahwaii, S. Ipson, \& AlFahdawi, S, "A Multimodal Biometric System For Personal Identification Based On Deep Learning Approaches" in Seventh International Conference on Emerging Security Technologies EST,2017, pp. 163168.

[10] E. Osherov, \& M. Lindenbaum, "Increasing CNN Robustness to Occlusions by Reducing Filter Support" in Proceedings of the IEEE International Conference on Computer Vision, 2017, pp. 550-561.

[11] D. Javale, C. A. Sashittal, S. Wakchaure, A. M. Phadnis, S. S. Patil, \& , R. S. Shahane, "A New Approach to Wireless Data Transmission Using Visible Light", in Fourth International Conference on Computing Communication Control and Automation,2018, pp. 1-4, ГICCUBEA].

[12] J. K. Xavier, N. Raveen, A. R. Aneesh \& M. V. Sarada, "Data Transfer Using Light Fidelity Technology", in International Journal of Pure and Applied Mathematics, vol. 119(15),2018, pp.903908.

[13] A.Sarkar, S. Agarwal \& A. Nath, "Li-Fi technologv: Data Transmission through Visible Light", in International Journal of Advance Research in Computer Science and Management Studies, vol. 3(6), 2015.

[14] S. Fairuz, M. H. Habaebi, E. M. A. Elsheikh, \& A. J. Chebil, "Convolutional Neural Network-based Finger Vein Recognition using Near Infrared Images" in 7th International Conference on Computer and Communication Engineering, pp. 453458ГICCCE 20181.

[15] E. L. Spratt, "Dream Formulations and Deep Neural Networks: Humanistic Themes in the Iconology of the Machine-Learned Image", 2018 「arXiv preprint arXiv: 1802.012741.

[16] Arthi. R, Jai Ahuia, Sachin Kumar, Pushpendra Thakur, Tanay Sharma, "Small Obiect Detection from Video and Classification using Deep Learning". In: Springer Lecture Notes in Electrical Engineering Advances in Systems, Control and Automations, Proceedings of ETAEERE-2020, Published online on March 2021, Vol 708, pp 100-107.

[17] C. Aravindan. R. Arthi, R. Kishankumar. V. Gokul, S. Giridaran, "A Smart Assistive System for Visually Impaired to inform acquaintance using Image Processing (ML) 
supported by IoT" for book titled "Hybrid Artificial Intelligence and IoT in Healthcare" with the Springer Premier Book Series "Intelligent Systems Reference Library" Vol: 209. PP 149-164

[18] Arthi. R, Allada Rahul Kishan. Aksa Abraham, Alekhya Sattenapalli "Centralized Intelligent Authentication System Using Deep Learning with Deep Dream Image Algorithm", In: Springer Lecture Notes in Electrical Engineering Advances in Power Systems and Energy Management. Proceedings of ETAEERE-2020, Published online on Jan 2021, Vol 690, pp.169-178.

[19] S. V. Reddy, K. T. Reddy, \& V. ValliKumari, "Optimization of Deep Learning Using Various Optimizers, Loss Functions and Dropout" in International Journal of Recent Technology Engineering vol.7,2018, pp.448-455.

\section{Creative Commons Attribution License 4.0 (Attribution 4.0 International, CC BY 4.0)}

This article is published under the terms of the Creative Commons Attribution License 4.0

https://creativecommons.org/licenses/by/4.0/deed.en US 